\title{
The Wavelength-shifting Optical Module for the IceCube Upgrade
}

\section{The IceCube Collaboration}

(a complete list of authors can be found at the end of the proceedings)

E-mail: jorackhe@uni-mainz.de, anna.pollmann@uni-wuppertal.de,

mrongen@uni-mainz.de

The Wavelength-shifting Optical Module (WOM) is a novel optical sensor that uses wavelength shifting and light guiding to substantially enhance the photosensitive area of UV optical modules. It has been designed for the IceCube Upgrade, a seven-string extension of the IceCube detector planned for the 2022/2023 South Pole deployment season. The WOM consists of a hollow quartz cylinder coated in wavelength shifting paint which serves as detection area and has two photomultipliers (PMTs) attached to the end faces. The light-collecting tube increases the effective photocathode area of the PMTs without producing additional dark current, making it suitable for low-signal, low-noise applications. We report on the design and performance of the WOM with a focus on the 12 modules in production for deployment in the IceCube Upgrade. While the WOM will be deployed in IceCube, its design is applicable to any large-volume particle detector based on the detection of Cherenkov light.

Corresponding authors: John Rack-Helleis ${ }^{1 *}$, Anna Pollmann ${ }^{2}$, Martin Rongen ${ }^{1}$

${ }^{1}$ Institute for Physics, JGU Mainz, D-55122 Mainz, Germany

${ }^{2}$ Department of Physics,Bergische Universität Wuppertal, Gaußstraße 20, Wuppertal, Germany

* Presenter

$37^{\text {th }}$ International Cosmic Ray Conference (ICRC 2021)

July 12th-23rd, 2021

Online - Berlin, Germany 


\section{The IceCube Neutrino Observatory and the IceCube Upgrade}

The IceCube Neutrino Observatory is a cubic-kilometer scale neutrino detector installed in the ice at the geographic South Pole [1]. Reconstruction of direction, energy, and flavor of the neutrinos relies on the optical detection of Cherenkov radiation emitted by charged particles produced in the interactions of neutrinos in the surrounding ice or the nearby bedrock.

The IceCube Upgrade [2] is planned to be deployed during the 2022/2023 South Pole Season and marks the first extension of the IceCube detector since its completion in 2010. Over 700 additional modules - the majority of which use the mDOM [3] and the D-Egg [4] designs - will be deployed on seven additional strings (chains of modules in one drill hole). The spacing between optical modules in the Upgrade will be $\sim 20 \mathrm{~m}$ horizontally and $3 \mathrm{~m}$ vertically, compared to $\sim 100 \mathrm{~m}$ horizontally and $17 \mathrm{~m}$ vertically in IceCube. This denser instrumentation lowers the energy threshold for neutrino detection, improves event reconstruction, and allows for a more precise calibration of the detector medium (the ice). Additionally, it will be used for in-situ studies of novel optical sensor designs. Among these will be the Wavelength-shifting optical module (WOM) of which we are preparing to deploy 12 units.

\section{The IceCube Upgrade WOM}

In order to instrument large detector volumes with photo-sensitive sensors capable to resolve single photons, the general approach is to use photomultiplier tubes in the detector modules. This means that the financial costs as well as the background noise rate scale linearly with the deployed sensitive area. By shifting the detection area from PMTs to a photon-capturing tube instead, the instrumented area is increased significantly by elongating the tube. The light captured by the tube can be focused on small photocathode areas, lowering the thermionic noise contribution in comparison to designs using PMTs only.
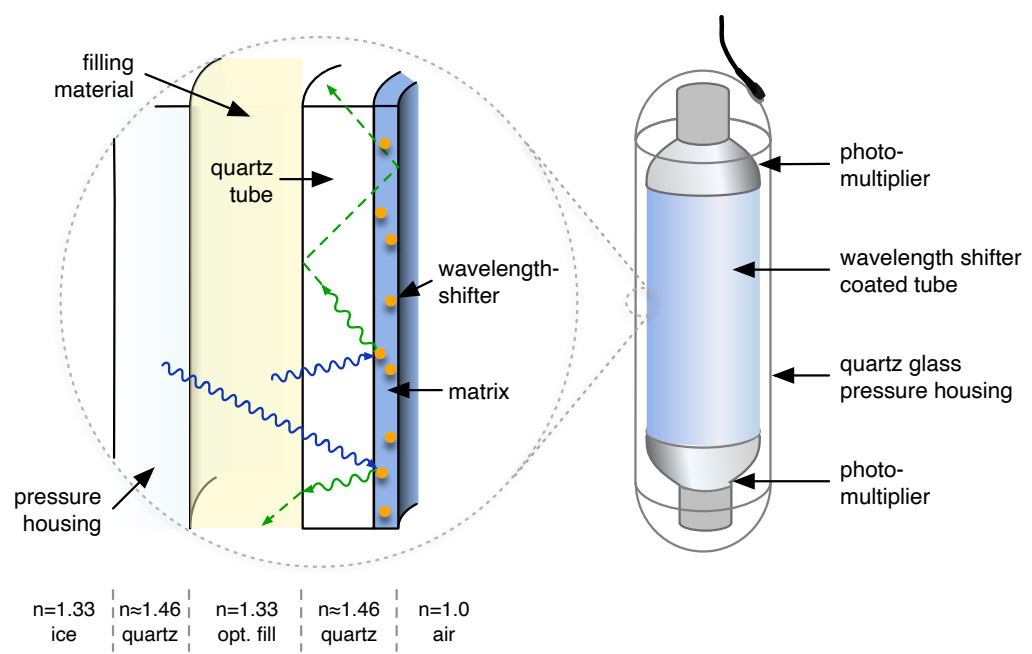

Figure 1: Sketch and working principle of the WOM. UV Photons are absorbed, shifted and reemitted by the wavelength shifting substrate. Reemitted photons are guided to the read-out PMTs by means of total internal reflection. 
The WOM, schematically shown in Figure 1, consists of a transparent tube which is coated with paint containing wavelength-shifting (WLS) organic luminophores. UV photons incident on the tube are absorbed in the paint layer with high efficiency and re-emitted isotropically at optical wavelengths.

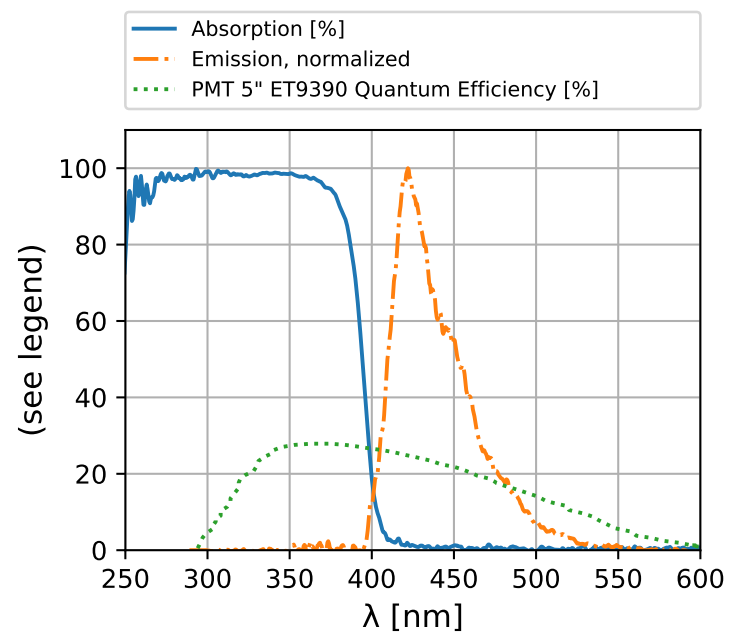

Figure 2: Absorption and emission profile of the WLS-paint in blue and orange. The quantum efficiency of the 5" ET9390 is shown in green.
Absorption and emission spectrum of the paint are shown in relation to the PMT quantum efficiency in Figure 2. If the photon emission angle is above the critical angle for total internal reflection, the photon is trapped in the tube and guided towards the read-out PMTs at the end faces. In comparison to the $\mathrm{mDOM}$ and the DEgg, the WOM offers two unique features: low overall noise rates, as the photocathode area is small in comparison to the sensitive area of the WLS tube, and enhanced UV sensitivity. The latter is especially desirable in IceCube, since the Cherenkov spectrum peaks in the UV range.

The WOM concept was first introduced in [5], the wavelength-shifting paint and the coating process have been thoroughly investigated in $[6,7]$, with testing procedures outlined in [8]. Here we discuss the specific developments required for the design and production of IceCube Upgrade WOMs. The choice of PMT and DAQ are discussed in section 3. The performance of a prototpye WLS tube is discussed in section 4. For the IceCube Upgrade, the WOM and the required electronics need to be assembled within a UV-transparent quartz housing serving as pressure vessel. This requirement as well as the mandatory filling material between the pressure housing and WLS tube are the content of section 5 and section 6. Assembly of all components is described in section 7 .

\section{Photomultiplier and data acquisition}

Since the diameter of the PMT constrains the diameters of the wavelength shifting tube as well as the pressure housing, the choice of PMT model is a critical design decision. The following requirements were considered:

- A reasonably large diameter to make best use of the available drill hole diameter

- Good efficiency at the edge of the photocathode, needed for coupling to the WLS tube

- Low thermionic noise to demonstrate good noise characteristics for supernova detection

- Gain $>5 \times 10^{6}$ at safe voltages, for single photon detection using the selected DAQ

- Flat cathode surface to ease gluing of the tube

Given these requirements, the Electron Tubes ET9330 ${ }^{1}$ was identified as the closest match and is currently under detailed investigation.

\footnotetext{
1https://et-enterprises.com/images/data_sheets/9390B.pdf
} 


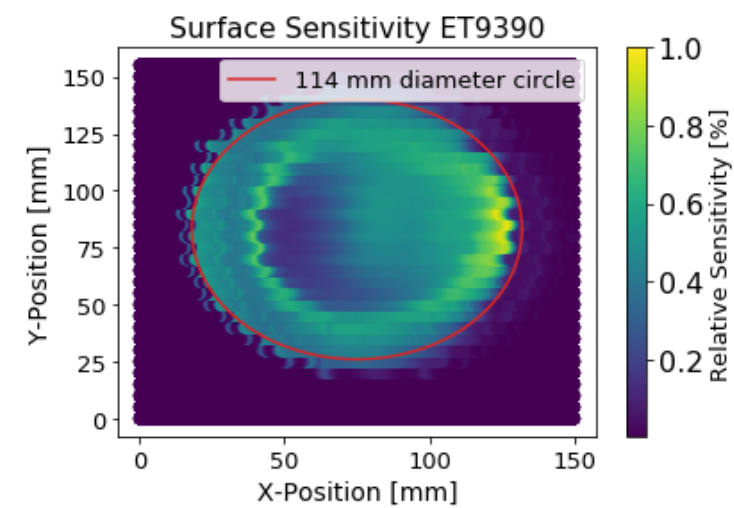

Figure 3: Relative surface sensitivity of the ET9390 Photocathode at $900 \mathrm{~V}$ supply voltage.

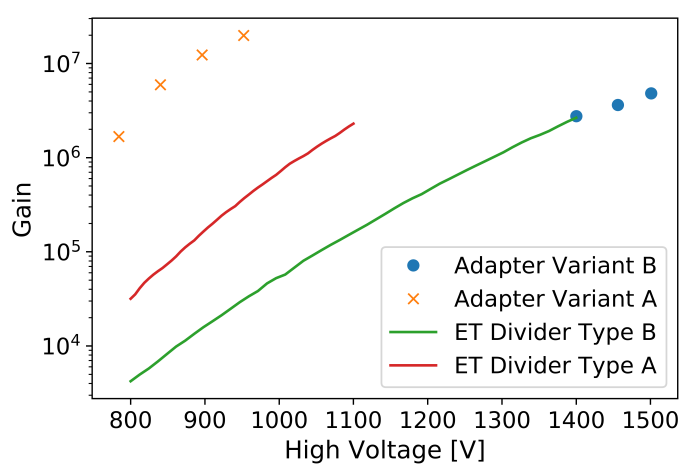

Figure 4: Gain measurement (dots and crosses) and data sheet values (lines) for different divider configurations.

In order to enable easy integration into the IceCube Upgrade computing and communication infrastructure, the analog signals from the PMTs will be read out using waveform micro-bases [9]. An IceCube Mini-Mainboard is used to handle communication on the string. The high voltage for the PMT is supplied using a staged Cockroft-Walton chain implemented on the waveform micro-base and as already used on the micro-bases for the mDOM 3" PMTs[3].

Custom adapter printed circuit board (PCBs) were developed to accommodate for the differences in pin-out and required voltage ratios. Following the manufacturer's recommendations, a low gain variant was successfully implemented (see Figure 4, labeled Adapter Variant B). Implementing the desired high gain configuration (similar to ET Divider Type A) has so far not been successful as the resulting single-photon charge distributions are very broad with no discernible valley.

Using the voltage ratios as directly available from the micro-base (3:1:1:1:1, see adapter variant A in Figure 4) results in a high gain of $10^{7}$ at a bias of $\approx 1000 \mathrm{~V}$, well below the recommended operating voltage. The resulting small potential between photocathode and first dynode is unfavorable as it is likely to result in an inhomogeneous collection efficiency and strong magnetic field dependencies. To gauge this effect, the surface sensitivity was measured using an attenuated pulsed laser mounted to a translation stage. The relative sensitivity map is shown in Figure 3. The photoactive area is approximately confined to a $114 \mathrm{~mm}$ diameter circle. The asymmetry in the inner diameter is attributed to the expected inhomogeneous collection efficiency caused by the low bias voltage. Figure 5 shows the PMT transit time spread using the adapter variant A. The standard deviation is $5.2 \mathrm{~ns}$ and thus smaller than the spread introduced in the WLS tube (see subsection 4.2). Further properties still to be studied include the PMT's thermionic noise as a function of temperature as well as dynamic range.

\section{Wavelength-shifting Tube}

The WLS-tube consists of quartz glass ${ }^{2}$ tube which is $76 \mathrm{~cm}$ long and $10.6 \mathrm{~cm}$ in outer diameter. The two 5" ET9330 PMTs and the WLS-tube are optically coupled using a UV curing glue ${ }^{3}$.

\footnotetext{
${ }^{2}$ https://www .heraeus.com/media/media/hca/doc_hca/products_and_solutions_8/solids/Solids_ HSQ300_330MF_EN.pdf

3https://www . norlandprod.com/adhesives/NOA146H.html
} 


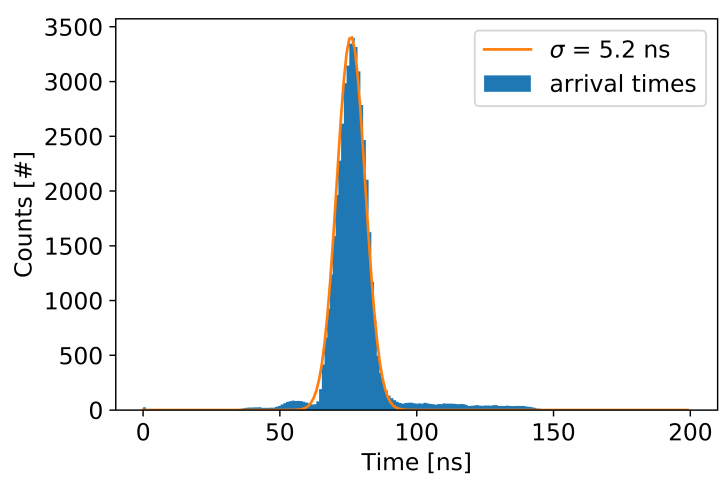

Figure 5: Response time spread of the Upgrade WOM PMT (ET9390).

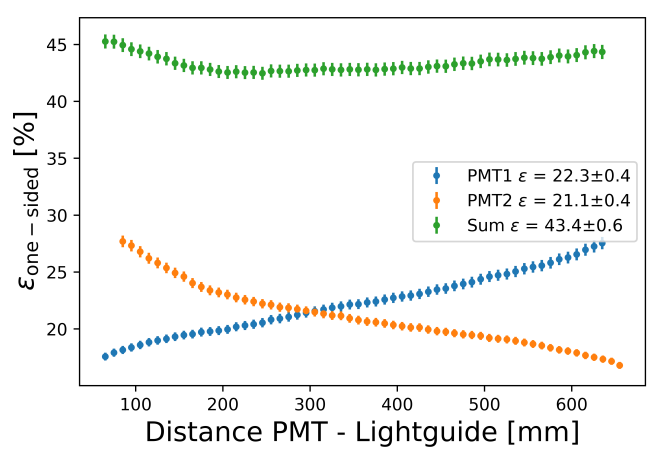

Figure 7: Light guiding efficiency of the inner Tube as a function of the $z$-coordinate. The orange and blue curves represent the individual efficiencies at the PMTs and green denotes the overall guiding efficiency.

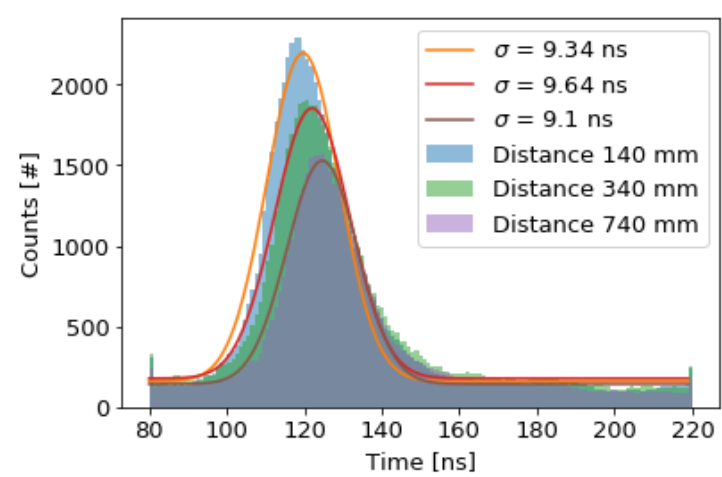

Figure 6: Photon arrival time distribution for selected $\mathrm{z}$ coordinates. The WLS tube has a length of $76 \mathrm{~cm}$

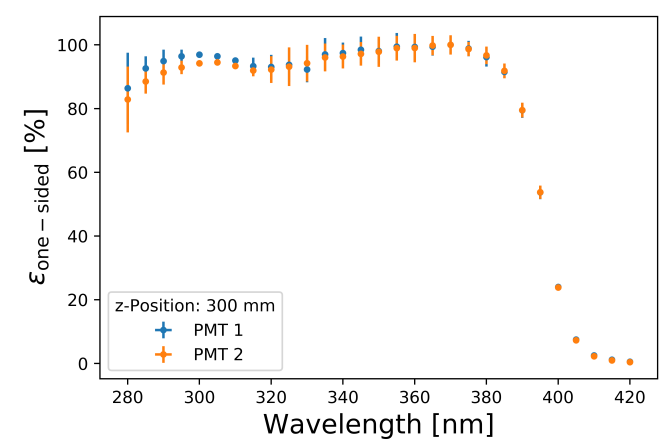

Figure 8: Capture efficiency of the WLS tube as a function of the incident light's wavelength. The measurement was performed in the middle of the tube and is corrected for transport losses.

\subsection{Efficiency}

To determine the WLS-tube efficiency as a function of distance along the tube (z-coordinate) and wavelength, a setup similar to the one described in [8] is used: The output of a xenon arc lamp is wavelength selected using a monochromator. The beam is chopped and its intensity is controlled using a photodiode read out by lock-in amplifiers. Using a movable liquid light guide allows for measuring the WOM's efficiency as a function of $z$ and $\lambda$. The efficiency dependence on the $z$-coordinate is shown in Figure 7 . It can be concluded that approximately $40 \%$ of the photons re-emitted by the WLS paint reach the readout PMTs. The measurement in Figure 8 shows that the process of absorption and re-emission of the wavelength-shifting paint has an efficiency of close to $100 \%$ in the wavelength range from $280 \mathrm{~nm}$ to $400 \mathrm{~nm}$.

\subsection{Timing}

The enhanced UV-sensitivity and low noise of the WOM come at the cost of a $2 \times$ wider distribution in photon arrival times compared to a bare PMT. Here, the major contribution is the travel time of the photons inside the WLS tube prior to reaching the PMTs. The absorption and 


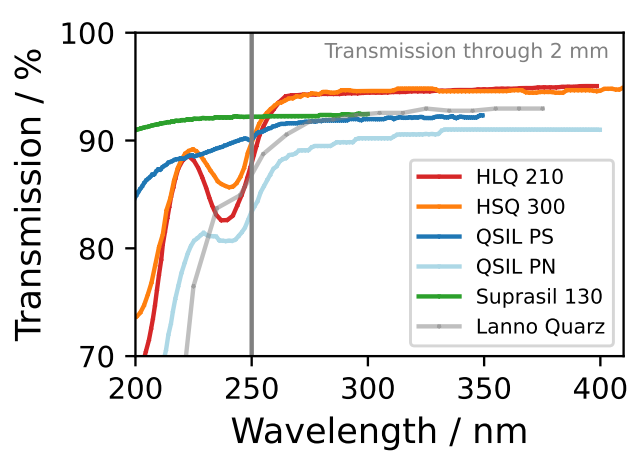

Figure 9: Measurement of the transmission of several glass samples considered for the pressure vessel. The interesting region lies above $250 \mathrm{~nm}$ ( grey line).

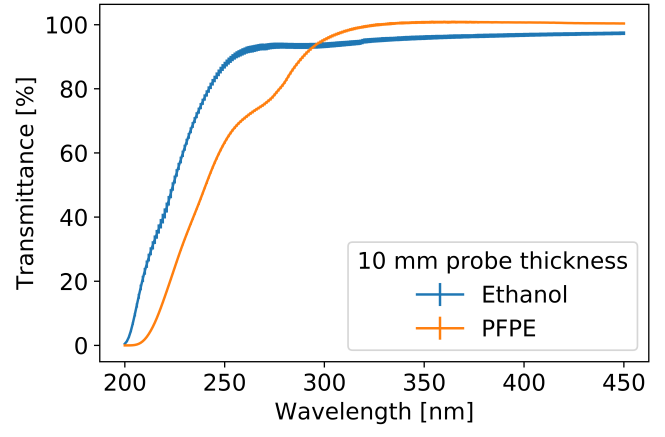

Figure 10: Transmission of selected filling materials for the upgrade WOM.

re-emission of the WLS paint plays a minor role due to its fast decay time of $1.35 \mathrm{~ns}$ [6]. The arrival time distribution of the WLS has been measured using a pico-second pulser[10] as light source and a standalone ADC to digitize the PMT signals. In Figure 6 the arrival time distribution for selected distances between the light entry point and readout PMT is shown. It can be observed, that for larger distances, the average arrival time shifts to later times, matching the time shift due to the effective speed of light in quartz glass. The overall timing resolution is approximated to be $10 \mathrm{~ns}$.

\section{Pressure Housing}

The choice of the pressure housing is critical, since low UV transmittance or background from the glass vessel due to radioactive impurities would significantly deteriorate the device's performance. In earlier prototypes, the isotope ${ }^{238} \mathrm{U}$ has been proven to cause significant background contribution.

Glass samples from several companies were investigated. The transparency was measured using a calibrated test stand and samples of different thickness. The results can be seen in Figure 9. The transmission above $250 \mathrm{~nm}$ exceeds $90 \%$ for almost all samples, rendering all shown options viable. For cost effectiveness reasons, the glass HLQ210 was chosen as material for the vessel.

Neutron activation was used to estimate the radioactive backgrounds in the glass to be approximately $6 \mathrm{~Bq} \mathrm{~kg}^{-1}$ amounting to a dark noise rate of $114 \mathrm{~Hz}$ for a $\sim 19 \mathrm{~kg}$ pressure vessel. This contribution is negligible compared to the lowest background expected from the PMTs ${ }^{4}$.

\section{Filling material}

The optimal filling material between the pressure vessel glass and the inner WLS tube was chosen based on two derivations: the effective area of the device was first calculated analytically accounting for all Fresnel transmissions as well as the total internal reflections in the WLS tube assuming homogeneous illumination with plane waves. Second, a raytracing Monte Carlo simulation was modeled taking these effects into account.

\footnotetext{
${ }^{4}$ https://et-enterprises.com/images/data_sheets/9390B.pdf
} 
Figure 11 shows the change in effective area as a function of the refractive index of the filling material. While the absolute difference between the approaches is still under investigation, they both demonstrate the optimum refractive index of the filling material is $n=1.33$. This opens up several choices for the filling material with Ethanol and PFPE (Perfluorpolyether) being the most prominent. We prefer PFPE over Ethanol due to the higher vapor pressure and chemical inertness. Its refractive index is $n=1.30$ and it shows good transparency in the UV, as shown in Figure 10.

\section{WOM Assembly}

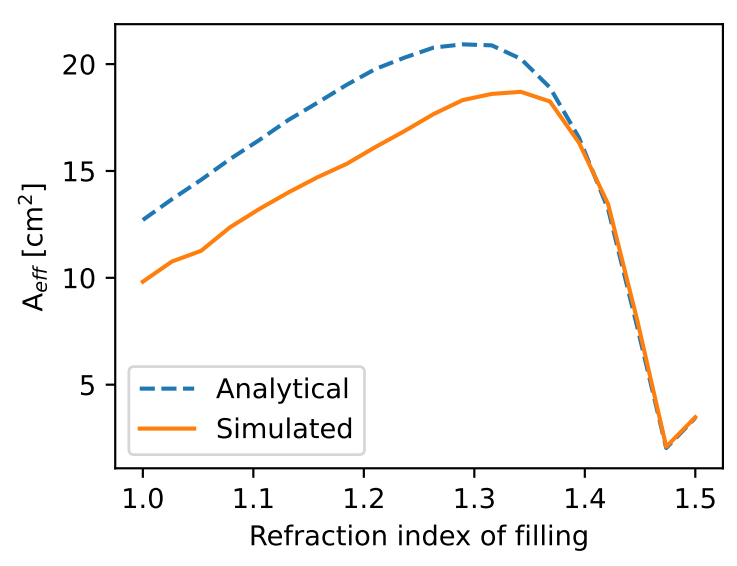

Figure 11: Effective area of the fully module as a function of the refractive index $n$ of the filling material obtained in two ways: an analytical derivation (blue) as well as a MC simulation of the device (orange). clamps on a load-bearing cable. One cable clamp acts as guide only, to allow the cable to extend $1 \%$ in length under load. In order to prevent vertical movement of the module, which slightly shrinks under pressure, metal bands are crossed above the end caps and welded to the pipe clamps.

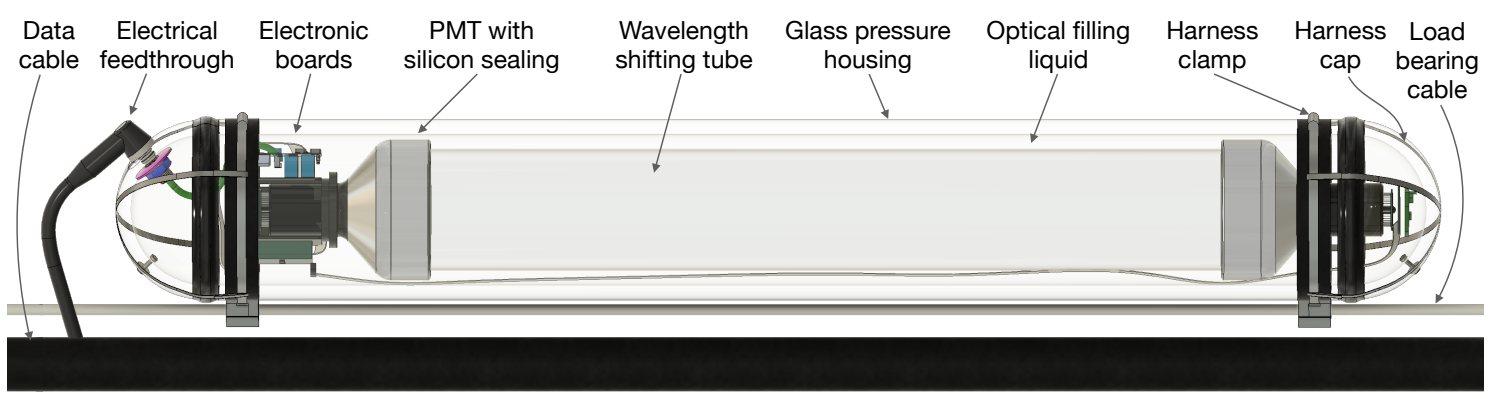

Figure 12: CAD drawing of the assembled WOM module.

The weight of the full module is mainly due to the approximately $19 \mathrm{~kg}$ of the vessel. The inner part consists of the WLS tube with attached PMTs, as described in section 4. The two PMTs

\footnotetext{
${ }^{5}$ https://www.heraeus.com/media/media/hca/doc_hca/products_and_solutions_8/solids/Solids_ HSQ300_330MF_EN.pdf
} 
are embedded in a silicone ${ }^{6}$ wrapper which matches the inner diameter of the pressure housing. The silicone confines the filling material ${ }^{7}$ to the space between the PMTs. Feedthrough holes for electronic cables as well as a pressure valve allow for pouring of the filling material and for pressure compensation during deployment.

\section{Summary and Outlook}

The Wavelength-shifting optical module is a UV-sensitive, low-noise photosensor which is being developed for the IceCube Upgrade. With this design, we obtain an effective area of approximately $19 \mathrm{~cm}^{2}$. It is most sensitive to photons between $280 \mathrm{~nm}$ to $400 \mathrm{~nm}$. The aim is to deploy the devices in clusters within the IceCube Upgrade array to locally lower the energy threshold, thus enhancing the resolution of low energy neutrino reconstructions. The reduced average noise also improves the sensitivity to supernova neutrinos, which are not individually resolvable but result in a small temporal rate increase over the rate of background events. The large and easily scalable effective area of the WOM in combination with the low inherent noise makes it interesting for other applications, e.g. instrumenting large veto volumes for particle detectors. The WOM is already incorporated into the design of the ShiP [11] experiment, based on a modified version without pressure vessel and using SiPMs instead of PMTs [12].

\section{References}

[1] IceCube Collaboration, M. G. Aartsen et al. JINST 12 no. 03, (2017) P03012.

[2] IceCube Collaboration, A. Ishihara PoS ICRC2019 (2021) 1031.

[3] IceCube Collaboration PoS ICRC2021 (these proceedings) .

[4] IceCube Collaboration PoS ICRC2021 (these proceedings) .

[5] L. Schulte et al. ICRC (2013) .

[6] D. Hebecker et al. PoS ICRC2015 (2016) 1134.

[7] D. Hebecker et al. EPJ Web of Conferences $116(01,2016) 01006$.

[8] IceCube-Gen2 Collaboration, P. Peiffer and D. Hebecker PoS ICRC2017 (2018) 1052.

[9] IceCube Collaboration PoS ICRC2021 (these proceedings) .

[10] M. Rongen and M. Schaufel JINST 13 no. 06, (2018) P06002.

[11] G. De Lellis Nuclear and Particle Physics Proceedings 263-264 (2015) 71-76. Capri 2014.

[12] M. e. a. Ehlert Journal of Instrumentation 14 no. 03, (Mar, 2019) P03021-P03021.

6https://wWw. smooth-on.com/tb/files/MOLD_STAR_15_16_30_TB.pdf

7https://www. solvay.com/en/product/galden-ht-170 


\section{Full Author List: IceCube Collaboration}

R. Abbasi ${ }^{17}$, M. Ackermann ${ }^{59}$, J. Adams ${ }^{18}$, J. A. Aguilar ${ }^{12}$, M. Ahlers ${ }^{22}$, M. Ahrens ${ }^{50}$, C. Alispach ${ }^{28}$, A. A. Alves Jr. ${ }^{31}$, N. M. $\mathrm{Amin}^{42}$, R. An ${ }^{14}$, K. Andeen ${ }^{40}$, T. Anderson ${ }^{56}$, G. Anton ${ }^{26}$, C. Argüelles ${ }^{14}$, Y. Ashida ${ }^{38}$, S. Axani ${ }^{15}$, X. Bai ${ }^{46}$, A. Balagopal V. ${ }^{38}$, A. Barbano ${ }^{28}$, S. W. Barwick ${ }^{30}$, B. Bastian ${ }^{59}$, V. Basu ${ }^{38}$, S. Baur ${ }^{12}$, R. Bay ${ }^{8}$, J. J. Beatty ${ }^{20}, 21$, K.-H. Becker ${ }^{58}$, J. Becker Tjus ${ }^{11}$, C. Bellenghi $^{27}$, S. BenZvi ${ }^{48}$, D. Berley ${ }^{19}$, E. Bernardini ${ }^{59,60}$, D. Z. Besson ${ }^{34,61}$, G. Binder ${ }^{8,9}$, D. Bindig ${ }^{58}$, E. Blaufuss ${ }^{19}$, S. Blot ${ }^{59}$, M. Boddenberg ${ }^{1}$, F. Bontempo ${ }^{31}$, J. Borowka ${ }^{1}$, S. Böser ${ }^{39}$, O. Botner ${ }^{57}$, J. Böttcher ${ }^{1}$, E. Bourbeau ${ }^{22}$, F. Bradascio ${ }^{59}$, J. Braun ${ }^{38}$, S. Bron $^{28}$, J. Brostean-Kaiser ${ }^{59}$, S. Browne ${ }^{32}$, A. Burgman ${ }^{57}$, R. T. Burley ${ }^{2}$, R. S. Busse ${ }^{41}$, M. A. Campana ${ }^{45}$, E. G. Carnie-Bronca ${ }^{2}$, C. $\mathrm{Chen}^{6}$, D. Chirkin ${ }^{38}$, K. Choi ${ }^{52}$, B. A. Clark ${ }^{24}$, K. Clark ${ }^{33}$, L. Classen ${ }^{41}$, A. Coleman ${ }^{42}$, G. H. Collin ${ }^{15}$, J. M. Conrad ${ }^{15}$, P. Coppin ${ }^{13}$, P. Correa ${ }^{13}$, D. F. Cowen ${ }^{55,}{ }^{56}$, R. Cross ${ }^{48}$, C. Dappen ${ }^{1}$, P. Dave ${ }^{6}$, C. De Clercq ${ }^{13}$, J. J. DeLaunay ${ }^{56}$, H. Dembinski ${ }^{42}$, K. Deoskar ${ }^{50}$, S. De Ridder $^{29}$, A. Desai ${ }^{38}$, P. Desiati ${ }^{38}$, K. D. de Vries ${ }^{13}$, G. de Wasseige ${ }^{13}$, M. de With ${ }^{10}$, T. DeYoung ${ }^{24}$, S. Dharani ${ }^{1}$, A. Diaz ${ }^{15}$, J. C. Díaz-Vélez ${ }^{38}$, M. Dittmer ${ }^{41}$, H. Dujmovic ${ }^{31}$, M. Dunkman ${ }^{56}$, M. A. DuVernois ${ }^{38}$, E. Dvorak ${ }^{46}$, T. Ehrhardt ${ }^{39}$, P. Eller ${ }^{27}$, R. Engel ${ }^{31,32}$, H. Erpenbeck ${ }^{1}$, J. Evans ${ }^{19}$, P. A. Evenson ${ }^{42}$, K. L. Fan ${ }^{19}$, A. R. Fazely ${ }^{7}$, S. Fiedlschuster ${ }^{26}$, A. T. Fienberg ${ }^{56}$, K. Filimonov ${ }^{8}$, C. Finley $^{50}$, L. Fischer ${ }^{59}$, D. Fox ${ }^{55}$, A. Franckowiak ${ }^{11,59}$, E. Friedman ${ }^{19}$, A. Fritz ${ }^{39}$, P. Fürst ${ }^{1}$, T. K. Gaisser ${ }^{42}$, J. Gallagher ${ }^{37}$, E. Ganster ${ }^{1}$, A. Garcia $^{14}$, S. Garrappa ${ }^{59}$, L. Gerhardt ${ }^{9}$, A. Ghadimi ${ }^{54}$, C. Glaser ${ }^{57}$, T. Glauch ${ }^{27}$, T. Glüsenkamp ${ }^{26}$, A. Goldschmidt ${ }^{9}$, J. G. Gonzalez ${ }^{42}$, S. Goswami ${ }^{54}$, D. Grant ${ }^{24}$, T. Grégoire ${ }^{56}$, S. Griswold ${ }^{48}$, M. Gündüz ${ }^{11}$, C. Günther ${ }^{1}$, C. Haack ${ }^{27}$, A. Hallgren ${ }^{57}$, R. Halliday ${ }^{24}$, L. Halve ${ }^{1}$, F. Halzen ${ }^{38}$, M. Ha Minh ${ }^{27}$, K. Hanson ${ }^{38}$, J. Hardin ${ }^{38}$, A. A. Harnisch ${ }^{24}$, A. Haungs ${ }^{31}$, S. Hauser ${ }^{1}$, D. Hebecker ${ }^{10}$, K. Helbing $^{58}$, F. Henningsen ${ }^{27}$, E. C. Hettinger ${ }^{24}$, S. Hickford ${ }^{58}$, J. Hignight ${ }^{25}$, C. Hill $^{16}$, G. C. Hill ${ }^{2}$, K. D. Hoffman ${ }^{19}$, R. Hoffmann ${ }^{58}$, T. Hoinka $^{23}$, B. Hokanson-Fasig ${ }^{38}$, K. Hoshina ${ }^{38,62}$, F. Huang ${ }^{56}$, M. Huber ${ }^{27}$, T. Huber $^{31}$, K. Hultqvist ${ }^{50}$, M. Hünnefeld ${ }^{23}$, R. Hussain ${ }^{38}$, S. In ${ }^{52}$, N. Iovine ${ }^{12}$, A. Ishihara ${ }^{16}$, M. Jansson ${ }^{50}$, G. S. Japaridze ${ }^{5}$, M. Jeong ${ }^{52}$, B. J. P. Jones ${ }^{4}$, D. Kang ${ }^{31}$, W. Kang ${ }^{52}$, X. Kang ${ }^{45}$, A. $\mathrm{Kappes}^{41}$, D. Kappesser ${ }^{39}$, T. Karg ${ }^{59}$, M. Karl ${ }^{27}$, A. Karle ${ }^{38}$, U. Katz ${ }^{26}$, M. Kauer ${ }^{38}$, M. Kellermann ${ }^{1}$, J. L. Kelley ${ }^{38}$, A. Kheirandish ${ }^{56}$, K. Kin ${ }^{16}$, T. Kintscher ${ }^{59}$, J. Kiryluk ${ }^{51}$, S. R. Klein ${ }^{8,9}$, R. Koirala ${ }^{42}$, H. Kolanoski ${ }^{10}$, T. Kontrimas ${ }^{27}$, L. Köpke ${ }^{39}$, C. Kopper ${ }^{24}$, S.

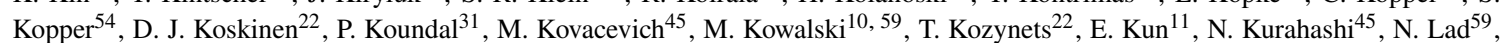
C. Lagunas Gualda ${ }^{59}$, J. L. Lanfranchi ${ }^{56}$, M. J. Larson ${ }^{19}$, F. Lauber $^{58}$, J. P. Lazar ${ }^{14}{ }^{38}$, J. W. Lee ${ }^{52}$, K. Leonard ${ }^{38}$, A. Leszczyńska ${ }^{32}$,

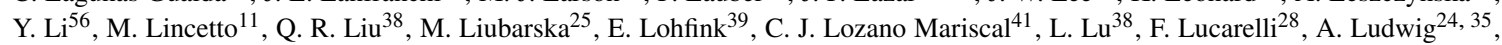
W. Luszczak ${ }^{38}$, Y. Lyu $^{8,9}$, W. Y. Ma ${ }^{59}$, J. Madsen ${ }^{38}$, K. B. M. Mahnn ${ }^{24}$, Y. Makino ${ }^{38}$, S. Mancina ${ }^{38}$, I. C. Mariş ${ }^{12}$, R. Maruyama ${ }^{43}$, K. Mase ${ }^{16}$, T. McElroy ${ }^{25}$, F. McNally ${ }^{36}$, J. V. Mead ${ }^{22}$, K. Meagher ${ }^{38}$, A. Medina ${ }^{21}$, M. Meier ${ }^{16}$, S. Meighen-Berger ${ }^{27}$, J. Micallef ${ }^{24}$,

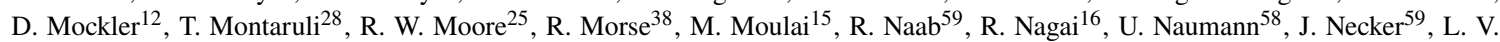
Nguyễn ${ }^{24}$, H. Niederhausen ${ }^{27}$, M. U. Nisa ${ }^{24}$, S. C. Nowickii ${ }^{24}$, D. R. Nygren ${ }^{9}$, A. Obertacke Pollmann ${ }^{58}$, M. Oehler ${ }^{31}$, A. Olivas ${ }^{19}$, E. O'Sullivan ${ }^{57}$, H. Pandya ${ }^{42}$, D. V. Pankova ${ }^{56}$, N. Park ${ }^{33}$, G. K. Parker ${ }^{4}$, E. N. Paudel ${ }^{42}$, L. Paul ${ }^{40}$, kkkC. Pérez de los $\operatorname{Heros}^{57}$, L. Peters ${ }^{1}$, J. Peterson ${ }^{38}$, S. Philippen ${ }^{1}$, D. Pieloth ${ }^{23}$, S. Pieper ${ }^{58}$, M. Pittermann ${ }^{32}$, A. Pizzuto ${ }^{38}$, M. Plum ${ }^{40}$, Y. Popovych ${ }^{39}$, A. Porcelli $^{29}$, M. Prado Rodriguez ${ }^{38}$, P. B. Price ${ }^{8}$, B. Pries ${ }^{24}$, G. T. Przybylski ${ }^{9}$, C. Raab ${ }^{12}$, A. Raissi ${ }^{18}$, M. Rameez ${ }^{22}$, K. Rawlins ${ }^{3}$, I. C. Rea ${ }^{27}$, A. Rehman ${ }^{42}$, P. Reichherzer ${ }^{11}$, R. Reimann ${ }^{1}$, G. Renzi ${ }^{12}$, E. Resconi ${ }^{27}$, S. Reusch ${ }^{59}$, W. Rhode ${ }^{23}$, M. Richman ${ }^{45}$, B. Riedel $^{38}$, E. J. Roberts ${ }^{2}$, S. Robertson ${ }^{8,9}$, G. Roellinghoff ${ }^{52}$, M. Rongen ${ }^{39}$, C. Rott ${ }^{49,52}$, T. Ruhe ${ }^{23}$, D. Ryckbosch ${ }^{29}$, D. Rysewyk $\mathrm{Cantu}^{24}$, I. Safa ${ }^{14,38}$, J. Saffer ${ }^{32}$, S. E. Sanchez Herrera ${ }^{24}$, A. Sandrock ${ }^{23}$, J. Sandroos ${ }^{39}$, M. Santander ${ }^{54}$, S. Sarkar ${ }^{44}$, S. Sarkar ${ }^{25}$, K. Satalecka $^{59}$, M. Scharf ${ }^{1}$, M. Schaufel ${ }^{1}$, H. Schieler ${ }^{31}$, S. Schindler ${ }^{26}$, P. Schlunder ${ }^{23}$, T. Schmidt ${ }^{19}$, A. Schneider ${ }^{38}$, J. Schneider ${ }^{26}$, F. G. Schröder ${ }^{31,42}$, L. Schumacher ${ }^{27}$, G. Schwefer ${ }^{1}$, S. Sclafani ${ }^{45}$, D. Seckel ${ }^{42}$, S. Seunarine ${ }^{47}$, A. Sharma ${ }^{57}$, S. Shefali ${ }^{32}$, M. Silva $^{38}$, B. Skrzypek ${ }^{14}$, B. Smithers ${ }^{4}$, R. Snihur ${ }^{38}$, J. Soedingrekso ${ }^{23}$, D. Soldin ${ }^{42}$, C. Spannfellner ${ }^{27}$, G. M. Spiczak ${ }^{47}$, C. Spiering ${ }^{59,}$, 61, J. Stachurska $^{59}$, M. Stamatikos ${ }^{21}$, T. Stanev ${ }^{42}$, R. Stein ${ }^{59}$, J. Stettner ${ }^{1}$, A. Steuer ${ }^{39}$, T. Stezelberger ${ }^{9}$, T. Stürwald ${ }^{58}$, T. Stuttard ${ }^{22}$, G. W. Sullivan ${ }^{19}$, I. Taboada ${ }^{6}$, F. Tenholt ${ }^{11}$, S. Ter-Antonyan ${ }^{7}$, S. Tilav ${ }^{42}$, F. Tischbein ${ }^{1}$, K. Tollefson ${ }^{24}$, L. Tomankova ${ }^{11}$, C. Tönnis ${ }^{53}$, S. Toscano $^{12}$, D. Tosi $^{38}$, A. Trettin ${ }^{59}$, M. Tselengidou ${ }^{26}$, C. F. Tung ${ }^{6}$, A. Turcati ${ }^{27}$, R. Turcotte ${ }^{31}$, C. F. Turley ${ }^{56}$, J. P. Twagirayezu ${ }^{24}$, B. $\mathrm{Ty}^{38}$, M. A. Unland Elorrieta ${ }^{41}$, N. Valtonen-Mattila ${ }^{57}$, J. Vandenbroucke ${ }^{38}$, N. van Eijndhoven ${ }^{13}$, D. Vannerom ${ }^{15}$, J. van Santen $^{59}, \mathrm{~S}$. Verpoest $^{29}$, M. Vraeghe ${ }^{29}$, C. Walck ${ }^{50}$, T. B. Watson ${ }^{4}$, C. Weaver ${ }^{24}$, P. Weigel ${ }^{15}$, A. Weindl ${ }^{31}$, M. J. Weiss ${ }^{56}$, J. Weldert ${ }^{39}$, C. Wendt $^{38}$, J. Werthebach ${ }^{23}$, M. Weyrauch ${ }^{32}$, N. Whitehorn ${ }^{24,35}$, C. H. Wiebusch ${ }^{1}$, D. R. Williams ${ }^{54}$, M. Wolf ${ }^{27}$, K. Woschnagg ${ }^{8}$, G. Wrede ${ }^{26}$, J. Wulff ${ }^{11}$, X. W. Xu $u^{7}$, Y. Xu ${ }^{51}$, J. P. Yanez ${ }^{25}$, S. Yoshida ${ }^{16}$, S. Yu ${ }^{24}$, T. Yuan ${ }^{38}$, Z. Zhang ${ }^{51}$

${ }^{1}$ III. Physikalisches Institut, RWTH Aachen University, D-52056 Aachen, Germany

${ }^{2}$ Department of Physics, University of Adelaide, Adelaide, 5005, Australia

${ }^{3}$ Dept. of Physics and Astronomy, University of Alaska Anchorage, 3211 Providence Dr., Anchorage, AK 99508, USA

${ }^{4}$ Dept. of Physics, University of Texas at Arlington, 502 Yates St., Science Hall Rm 108, Box 19059, Arlington, TX 76019, USA

${ }^{5}$ CTSPS, Clark-Atlanta University, Atlanta, GA 30314, USA

${ }^{6}$ School of Physics and Center for Relativistic Astrophysics, Georgia Institute of Technology, Atlanta, GA 30332, USA

${ }^{7}$ Dept. of Physics, Southern University, Baton Rouge, LA 70813, USA

${ }^{8}$ Dept. of Physics, University of California, Berkeley, CA 94720, USA

${ }^{9}$ Lawrence Berkeley National Laboratory, Berkeley, CA 94720, USA

${ }^{10}$ Institut für Physik, Humboldt-Universität zu Berlin, D-12489 Berlin, Germany

${ }^{11}$ Fakultät für Physik \& Astronomie, Ruhr-Universität Bochum, D-44780 Bochum, Germany

${ }^{12}$ Université Libre de Bruxelles, Science Faculty CP230, B-1050 Brussels, Belgium

${ }^{13}$ Vrije Universiteit Brussel (VUB), Dienst ELEM, B-1050 Brussels, Belgium

${ }^{14}$ Department of Physics and Laboratory for Particle Physics and Cosmology, Harvard University, Cambridge, MA 02138, USA

${ }^{15}$ Dept. of Physics, Massachusetts Institute of Technology, Cambridge, MA 02139, USA 
${ }^{16}$ Dept. of Physics and Institute for Global Prominent Research, Chiba University, Chiba 263-8522, Japan

${ }^{17}$ Department of Physics, Loyola University Chicago, Chicago, IL 60660, USA

${ }^{18}$ Dept. of Physics and Astronomy, University of Canterbury, Private Bag 4800, Christchurch, New Zealand

${ }^{19}$ Dept. of Physics, University of Maryland, College Park, MD 20742, USA

${ }^{20}$ Dept. of Astronomy, Ohio State University, Columbus, OH 43210, USA

${ }^{21}$ Dept. of Physics and Center for Cosmology and Astro-Particle Physics, Ohio State University, Columbus, OH 43210, USA

${ }^{22}$ Niels Bohr Institute, University of Copenhagen, DK-2100 Copenhagen, Denmark

${ }^{23}$ Dept. of Physics, TU Dortmund University, D-44221 Dortmund, Germany

${ }^{24}$ Dept. of Physics and Astronomy, Michigan State University, East Lansing, MI 48824, USA

${ }^{25}$ Dept. of Physics, University of Alberta, Edmonton, Alberta, Canada T6G 2E1

${ }^{26}$ Erlangen Centre for Astroparticle Physics, Friedrich-Alexander-Universität Erlangen-Nürnberg, D-91058 Erlangen, Germany

${ }^{27}$ Physik-department, Technische Universität München, D-85748 Garching, Germany

${ }^{28}$ Département de physique nucléaire et corpusculaire, Université de Genève, CH-1211 Genève, Switzerland

${ }^{29}$ Dept. of Physics and Astronomy, University of Gent, B-9000 Gent, Belgium

${ }^{30}$ Dept. of Physics and Astronomy, University of California, Irvine, CA 92697, USA

${ }^{31}$ Karlsruhe Institute of Technology, Institute for Astroparticle Physics, D-76021 Karlsruhe, Germany

${ }^{32}$ Karlsruhe Institute of Technology, Institute of Experimental Particle Physics, D-76021 Karlsruhe, Germany

${ }^{33}$ Dept. of Physics, Engineering Physics, and Astronomy, Queen's University, Kingston, ON K7L 3N6, Canada

${ }^{34}$ Dept. of Physics and Astronomy, University of Kansas, Lawrence, KS 66045, USA

${ }^{35}$ Department of Physics and Astronomy, UCLA, Los Angeles, CA 90095, USA

${ }^{36}$ Department of Physics, Mercer University, Macon, GA 31207-0001, USA

${ }^{37}$ Dept. of Astronomy, University of Wisconsin-Madison, Madison, WI 53706, USA

${ }^{38}$ Dept. of Physics and Wisconsin IceCube Particle Astrophysics Center, University of Wisconsin-Madison, Madison, WI 53706, USA

${ }^{39}$ Institute of Physics, University of Mainz, Staudinger Weg 7, D-55099 Mainz, Germany

${ }^{40}$ Department of Physics, Marquette University, Milwaukee, WI, 53201, USA

${ }^{41}$ Institut für Kernphysik, Westfälische Wilhelms-Universität Münster, D-48149 Münster, Germany

42 Bartol Research Institute and Dept. of Physics and Astronomy, University of Delaware, Newark, DE 19716, USA

${ }^{43}$ Dept. of Physics, Yale University, New Haven, CT 06520, USA

${ }^{44}$ Dept. of Physics, University of Oxford, Parks Road, Oxford OX1 3PU, UK

${ }^{45}$ Dept. of Physics, Drexel University, 3141 Chestnut Street, Philadelphia, PA 19104, USA

${ }^{46}$ Physics Department, South Dakota School of Mines and Technology, Rapid City, SD 57701, USA

${ }^{47}$ Dept. of Physics, University of Wisconsin, River Falls, WI 54022, USA

${ }^{48}$ Dept. of Physics and Astronomy, University of Rochester, Rochester, NY 14627, USA

${ }^{49}$ Department of Physics and Astronomy, University of Utah, Salt Lake City, UT 84112, USA

50 Oskar Klein Centre and Dept. of Physics, Stockholm University, SE-10691 Stockholm, Sweden

${ }^{51}$ Dept. of Physics and Astronomy, Stony Brook University, Stony Brook, NY 11794-3800, USA

52 Dept. of Physics, Sungkyunkwan University, Suwon 16419, Korea

${ }^{53}$ Institute of Basic Science, Sungkyunkwan University, Suwon 16419, Korea

${ }^{54}$ Dept. of Physics and Astronomy, University of Alabama, Tuscaloosa, AL 35487, USA

${ }^{55}$ Dept. of Astronomy and Astrophysics, Pennsylvania State University, University Park, PA 16802, USA

56 Dept. of Physics, Pennsylvania State University, University Park, PA 16802, USA

${ }^{57}$ Dept. of Physics and Astronomy, Uppsala University, Box 516, S-75120 Uppsala, Sweden

${ }^{58}$ Dept. of Physics, University of Wuppertal, D-42119 Wuppertal, Germany

${ }^{59}$ DESY, D-15738 Zeuthen, Germany

${ }^{60}$ Università di Padova, I-35131 Padova, Italy

${ }^{61}$ National Research Nuclear University, Moscow Engineering Physics Institute (MEPhI), Moscow 115409, Russia

${ }^{62}$ Earthquake Research Institute, University of Tokyo, Bunkyo, Tokyo 113-0032, Japan

\section{Acknowledgements}

USA - U.S. National Science Foundation-Office of Polar Programs, U.S. National Science Foundation-Physics Division, U.S. National Science Foundation-EPSCoR, Wisconsin Alumni Research Foundation, Center for High Throughput Computing (CHTC) at the University of Wisconsin-Madison, Open Science Grid (OSG), Extreme Science and Engineering Discovery Environment (XSEDE), Frontera computing project at the Texas Advanced Computing Center, U.S. Department of Energy-National Energy Research Scientific Computing Center, Particle astrophysics research computing center at the University of Maryland, Institute for Cyber-Enabled Research at Michigan State University, and Astroparticle physics computational facility at Marquette University; Belgium - Funds for Scientific Research (FRS-FNRS and FWO), FWO Odysseus and Big Science programmes, and Belgian Federal Science Policy Office (Belspo); Germany - Bundesministerium für Bildung und Forschung (BMBF), Deutsche Forschungsgemeinschaft (DFG), Helmholtz Alliance for Astroparticle Physics (HAP), Initiative and Networking Fund of the Helmholtz Association, Deutsches Elektronen Synchrotron (DESY), and High Performance Computing cluster of the RWTH Aachen; Sweden - Swedish Research Council, Swedish Polar Research Secretariat, Swedish National Infrastructure for Computing (SNIC), and Knut and Alice Wallenberg Foundation; Australia - Australian 
Research Council; Canada - Natural Sciences and Engineering Research Council of Canada, Calcul Québec, Compute Ontario, Canada Foundation for Innovation, WestGrid, and Compute Canada; Denmark - Villum Fonden and Carlsberg Foundation; New Zealand Marsden Fund; Japan - Japan Society for Promotion of Science (JSPS) and Institute for Global Prominent Research (IGPR) of Chiba University; Korea - National Research Foundation of Korea (NRF); Switzerland - Swiss National Science Foundation (SNSF); United Kingdom - Department of Physics, University of Oxford. 